\title{
Congestion Control Techniques in a Computer Network: A Survey
}

\author{
Mirza Waseem Hussain \\ Department of Computer \\ Science, \\ Baba Ghulam Shah Badshah \\ University, Rajouri, J\&K, India.
}

\author{
Sanjay Jamwal, Ph.D. \\ Department of Computer \\ Science, \\ Baba Ghulam Shah Badshah \\ University, Rajouri, J\&K, India
}

\author{
Majid Zaman, Ph.D. \\ Directorate of Information \\ Technology \& Support System \\ University of Kashmir, \\ Hazratbal, Srinagar, J\&K, India
}

\begin{abstract}
With the advance in technology the focus has shifted from desktops and laptops to handheld devices like tablets, phablets, mobile phones, PDA etc. giving rise in the number of users connected to internet. About 900 million computers are connected to internet [23].In an hour 383 thousand TB of data transmission takes place [24]. As the traffic on internet increases giving rise to the problem of congestion. Several congestion techniques have been proposed, in this paper the summary of several congestion techniques and methods over the years has been discussed. One of the latest approaches to control the congestion is based on Neural Networks is also included in this paper
\end{abstract}

\section{Keywords}

Congestion control, TCP, Neural Networks, ECN, XCN, ATM, MANETS, EWCCP, Back propagation algorithm.

\section{INTRODUCTION}

A Network is said to be in the state of congestion when the total demands for resources exceeds the total available resources .In other words we can say a network is in a state of congestion when the quality of the service delivered to the user decreases, whenever there is increase of load in a network. Take an example of downloading a 1 GB file. When there is no congestion, the file gets downloaded in few minutes, but on day when there is congestion in a network same file gets downloaded in hours. The problem of congestion has become an important issue these days.

\section{BACKGROUND}

In the year 1999 Saverio Mascolo in his paper titled [21] states that there is a large bandwidth delay products in high speed communication networks thus resulting in adverse conditions on closed-loop congestion control algorithm. By using the combination of classical theory and Smiths principle a law for congestion control for high speed communication networks can be designed. This control law is transformed into discrete values and window form for ATM and internet respectively thus guarantying full and stable utilization of queue, links during transit and steady phase.

In the year 2001 Deepak Bansal And Hari Balakrishnan in their paper titled [6] states that congestion results in reduction of transmission rate that is problematic for voice and video applications ,therefore an algorithm that is nonlinear in nature is needed naming this algorithm as binomial Algorithm. The Binomial algorithm makes use of TCP style for Addictive increase and multiple decrease for increasing and decreasing the transmission rate. In addictive increase the rate of increase is inversely proportional to power $\mathrm{p}$ of current window size and for multiple decrease, rate of decrease is proportional to power $\mathrm{z}$ of current window size. There can be infinite number of such binomial algorithms, those that satisfy $\mathrm{p}+\mathrm{z}=1$ and all binomial algorithms converges for the fairness.
In the year $2002 \mathrm{~J} . A$ lan Bivens in his paper titled [10] predicts the congestion in a network using neural network learning technique, in which neural networks are used to predict the source of congestion, the system has been divided into three programs, one implementation consists of $\mathrm{c}$ language used for manipulation of file, the language used for running simulation and the one used for implementation in Tcl. The communication between the programs is done via files, the simulated network consists of six sending nodes connected to single receiving node where the packets are directed to their destination. During simulation the links are been monitored and nodes data is written onto respective files and these files are used for performing bulk computations using $\mathrm{c}$ wrapper for Neural network program. The mask representation which is the output of neural network is used for detecting source responsible for congestion.

In the same year work of Dina Katabi, Mark Handley and Charlie Rohrs in their paper [5] shows that product of latency and bandwidth, increases for per flow using theory and experiments as such instability inefficiency is increased regardless of size of queuing scheme in TCP. The TCP is outperformed by introducing a new protocol called as explicit congestion protocol $(\mathrm{XCN})$ that is generalization of explicit congestion notification protocol (ECN).Aggressiveness of the system is adjusted based on feedback delay, bandwidth that is spare and the reallocation of bandwidth flow is done if utilization of bandwidth is above the fair share. Here instead of sending one bit information to the sender, the sender is informed about the degree of congestion at the bottleneck therefore allowing the sender to adjust the window size. Providing high link utilization and efficient bandwidth allocation.

Aloke Chaudhuri in the same year quotes in his patent [1] that the computational properties of neural networks can be used for selecting the optimal route in a communication network. The traffic management and selection of optimal route are based on the output measurements of the network. Links in a network are monitored to obtain the historical data. This historical data is used to train the neural networks for predicting the best link by taking into consideration the factors like quality of service, cell rate and cost of the link. The prediction information along with topology information is provided to route discovery engine for processing, to select the best link for the communication.

In the same year Pallapa Venkataram, Sudip Ghosal and B.P. Vijay Kumar added to the pool of knowledge and quotes in their paper titled as [20] the parallel computational nature of neural network can be utilized for solving the optimization problem. Using this optimization problem for routing algorithm called as flow deviation algorithm, with the goal of minimizing average delay in a packet switching network. The algorithm uses recurrent neural network for finding the 
shortest path from source to destination thus providing optimal path with less average delay time.

In the year 2003 L. Khoukhi and S. Cherkaoui in their paper titled [14] states for ad hoc networks, congestion control mechanism is done by using intelligent solutions and uses the fuzzy logics to improve congestion control .The author in this paper presents two techniques. The first one is used for performing threshold management of buffer with the help of fuzzy logic system and the second uses Fuzzy Petri Nets for analysing to make decisions for congestion control.

In the year 2004 Lisong $\mathrm{Xu}$, Khaled Harfoush, and Injong Rhee states in their paper titled [13] as the TCP is unable to utilize the full capacity of network for high speed networks striving for new approach. In this approach initially unfairness of round trip time for existing protocols is identified and then a new scheme for congestion is used that alleviates the unfairness of round trip time problem while maintaining TCP friendly behaviour and scalability of bandwidth. The algorithm makes use of two window control policies. First one is used when window size is large ensuring square of round trip time unfairness while supporting scalability and the second one binary search increase that is been used when the size of window is small ensuring friendly behaviour of TCP

In the same year it was Lijun Chen, Steven H. Low and John C. Doyle in their paper titled as [12] and presents a unified design for ad hoc wireless networks by combining the design for congestion control and media access control. The allocation of resources are maximized by using contention matrix and contention graph while taking into account the contention that arises due to channel access. Two algorithms are presented that decomposes vertically into two protocol layers. The first one is called as primal algorithm that is used by the MAC layer to generate the price of congestion based on local aggregate rate and this local aggregate rate is utilized by TCP source to adjust the rate. Second one is called as Dual subgradient algorithm where the price of link is taken into consideration for implementation through scheduling link layer.

In the year 2005 work of Hyun C. Cho in his paper titled as [9] shows that the Back-Propagation (BP) algorithm of neural network can be used for technique of Active Queue Management. The dynamic nature of neural network is utilized to perform as a robust adaptive feedback controller after providing sufficient and necessary training data for adequate time period. The control can easily adapt to dynamic changing nature of TCP networks. The neural network calculates the probability and using this probability to determine the window size of TCP source .Neural networks minimize the error signal and are able to adjust AQM performance parameters to their optimal values. The size of actual queue is regulated very close to reference level after performing adequate training, the system works as adaptive controller under dynamic changing conditions.

In the same year Fernando Paganini, Zhikui Wang, John C. Doyle and Steven H. Low in their paper titled [7] states that the main aim of this paper is to design a congestion control system that is able to fully utilize the network resources by providing scalability, high utilization of network resources, low queuing delay, highly dynamic stability and fair share among the users. In this paper two types of control laws are developed. The first one called as dual control law that is able to scale gracefully with the capacity of the network, provide high resource utilization and low queuing delay but with constrained resource allocation policy. The second one called as primal dual overcoming the limitations of dual, further for communicating congestion message from links to source the protocols are implemented at two levels using queuing delay and ECN marking.

In the year 2006 C. Chrysostomou, A Pitsillides, G. Hadjipollas, A.Sekercioglu and M. Polycarpou quotes in their paper titled [4] that we can make use of fuzzy logic approach for active queue management (AQM) scheme and use explicit congestion notification to predict the congestion in a network. The packets in TCP/IP network can be marked using linguistic rules and buffer of router queue are operated on by fuzzy inference engine. The system makes the use of multiple inputs for capturing the state of a network and for capturing the dynamics of nonlinear probability marking function the system makes use of linguistic knowledge.

In the same Yen Chieh Ouyang, Ching-Wen Yang and Wei Shi Lian published their work on the paper titled [22]. In this paper the feedback rate regulator is used by applying the technique of neural networks for ATM networks in which the prediction of incoming load is made. Multiple leaky buckets are used for multiple sources and these leaky buckets can be utilized to monitor the source. Multiple leaky buckets (MLB) for variable bit rate can be used to predict the load of the traffic. The capacity of buffer and rate of leak can be adjusted. Incoming traffic load can be predicted by using finite duration impulse response (FIR) and this information gathered is passed to feedback rate regulator. The output of FIR multilayer provides the necessary information for discarding the cell. Therefore making high utilization of bandwidth while decreasing the cell loss rate.

In the same year Chonggang Wang, Kazem Sohra, Victor Lawrence,Bo Li, and Yueming Hu quotes in their paper titled [3] as in wireless sensor networks the nodes are densely distributed and produces high flow rate at the sink due to converging nature of upstream traffic. This results in congestion thus affecting the throughput and wastes energy due to packet loss. Therefore congestion is needed to be controlled for high utilization of resources, preventing low delay time and providing quality of service. Therefore suggesting a node priority based congestion control protocol (PCCP). The importance of a node is reflected by introducing a node priority index. The congestion degree is measured using packet arrival time along with packet service time, using this congestion degree and node priority index to impose hop to hop control.

Nenad Kojic ,Irini Reljin, and Branimir Reljin in the same year in their paper titled[18] suggests an efficient neural network based algorithm for optimizing the routing in a network .The high computational capabilities of neural network and ability to work on uncertain data are utilized for finding the optimal route in a network. Hopfield neural network is used for finding the optimal (shortest) path in a communication network taking into consideration incoming traffic flow, load of router, capacity of link thus preventing the loss of packets.

In the year 2007 the work of Kun Tan, Feng Jiang, Qian Zhang and Xuemin Shen in their paper titled [11] show that for multihop wireless networks, the unique characteristics of shared media possess a limitation of performance for conventional congestion control protocol such as TCP. On the other hand lack of coordination between nodes and imprecise congestion indication results in failure of TCP to utilize the allocated resources. As such a novel Explicit Wireless Congestion Control Protocol (EWCCP) is presented for 
stationary multihop wireless networks. EWCCP is able to gain fine grain control, dynamic robustness by exploiting multi bit explicit feedback and explicit coordination from routers. EWCCP is stabilized at smaller size but is able to send size of a window as compared to TCP resulting in low buffer occupancy and low delay. EWCCP is able to allocate a fair share of resources between the flows competing for the shared media.

In the year 2008 Hyun Cheol Cho, M. Sami Fadali and Kwon Soon Lee in their paper titled [8] presents a dynamic neural network approach for active queue management. Neural networks can with stand the non-linear time varying nature and stochastic nature of TCP. The recurrent neural networks are trained to regulate the actual size of the queue close to reference value of a queue. The recurrent neural networks work as an adaptive and robust controller thus providing an excellent performance even if the conditions are not included in training set.

In the year 2011 Melinda Barbas, Georgeta Boanea and Virgil Dobrota in their paper [16] states the use of forecasting algorithm for predicting the traffic. The main goal of this work is to separate the monitoring phase from routing process. Monitoring of links and communication between neighbouring nodes is done by routing management system. While routing decision and packet forwarding is done by multipath routing algorithm. The multipath routing algorithm in this paper is called as SAMP (Situation Aware Multipath). The SAMP uses the status information about the network provided by routing management system. Load balancing is employed for efficient utilization of the link by dividing the traffic among multiple routes and using split granularity at flow level to overcome the problem of out of order arrival of packets. If the congestion occurs the traffic is rerouted one by one until congestion is avoided. The routing management system is a dynamically self-managing system that is capable of adapting to changing external events.

In the year 2012 the work of Mehdi Mohtashamzadeh and Mehdi Harizi in their paper titled [15] shows, the neural network can be used to predict the congestion in a network before the quality of service degrades. The neural network system is divided into sub neural networks to deduce the complexity of the system and to enhance the computational capabilities by making the subparts to work in parallel. The first subsystem is two time-scale congestion and the second one multipart neural controller. The two time scale controller is used for performing congestion control in two stages, short time scale (20 -200ms) and long-time scales (3 second). For short time scales New Reno is used in which the size of the window is increased linearly after the threshold value is achieved. The second part is used for making decisions compatible for the long time decisions. The multiple feed forward neural network predicts the status of the network. At the end of the time scale neural network comes into play for predicting level of traffic. To speed up the overall communication process the proposed method is only used on selected nodes and routers.

The work of P.Rama Devi and Dr .D. Srinivasa Rao in the same year in their paper titled [19] proposes a protocol for balancing the load in MANET using congestion adaptive multipath routing protocol. When the source node wants to forward data to destination it makes use of reactive discovery technique and multiple path are established using Dijkstra algorithm. If there occurs congestion in the discovered route the congestion message is sent to the source. Upon receiving the congestion message by the source, the source distributes the packets through the cached routes using random transmission. If the route is not available in the cached list then it re-establishes the route and distributes the traffic, hence balancing the load in a network and preventing congestion.

In the year 2013 N. kojic, I.Reljin and B. Reljin states in their paper titled [17] that under arbitrary topology and under variable traffic conditions for Dynamic multicast routing the Hopfield neural network is used. The algorithm makes uses of information about the network parameter and historical information about links, routers. The main goal of this algorithm is to find optimal path for multicast routing. The Hopfield neural network energy function is used which includes the parameters like link distance, link delay, and bandwidth to find optimal path.

In the year 2014 B.Subramani and Dr. T. Karthikeyan in their paper titled as [2] states that for wireless communication the loss of packets is much more than that of wired communication due network congestion, thus proposing a mechanism to control congestion and naming it as Expertcontrol based intelligent multicast congestion control (ECMCC).The ECMCC uses the packet loss, queuing delay and feedback information to multicast source for detecting the congestion state in a network, thus distinguishing between the network congestion and error in wireless links thus taking different control strategies for controlling the rate at the source. The radial basis function neural network generates the knowledge base. Then this knowledge base is used by expert controller to control the congestion.

\section{CONCLUSION}

This paper discusses the various techniques for controlling the congestion in a network. With the increase in data transmission the number of challenges and issue comes into play, one of the major issue due to heavy traffic is the congestion. To control the congestion various techniques and methods have been evolved time and again. Designing the perfect technique for controlling the congestion in a network is very difficult. Hence the survey for various techniques for controlling congestion is very important. The techniques for congestion control are generally based on queue management and path routing. Several protocols have been devised to control the congestion. With the passage of time, it was found that some intelligent techniques are required to solve this problem of congestion. Therefore to solve this problem one of the approach is neural network, due to their ability to learn, fault tolerant nature, dynamic and high computational nature. In future we will use neural networks for finding the best route from source to destination in a network. The ultimate goal of the congestion control is to make optimal utilization of network resources. Therefore congestion must be avoided as it leads to degrade in the performance of the network.

\section{REFERENCES}

[1] Aloke Chaudhuri, "Route Optimization and Traffic Management In An ATM Network using Neural Network”, in United States Patent ,Jun 252002

[2] B.Subramani and Dr. T. Karthikeyan,“ Intelligent Multicast Congestion Based on Expert-Control with RBFNN for Wireless Networks", in International Journal Computer Technology and Applications, Vol. 5 (2), 375 382 March-April 2014

[3] Chonggang Wang, Kazem Sohra, Victor Lawrence, Bo $\mathrm{Li}$, Yueming $\mathrm{Hu}$," Priority-based Congestion Control in 
Wireless Sensor Networks", in Proceedings of the IEEE International Conference on Sensor Networks, Ubiquitous, and Trustworthy Computing (SUTC'06), 2006

[4] C. Chrysostomou, A Pitsillides, G. Hadjipollas, A.Sekercioglu and M. Polycarpou, "Fuzzy Logic Congestion Control In TCP/IP Best-Effort Networks" 2006

[5] Dina Katabi, Mark Handley Charlie Rohrs, "Congestion Control for High Bandwidth Delay Product Networks", Pittsburgh, Pennsylvania, USA SIGCOMM, 2002.

[6] Deepak Bansal And Hari Balakrishnan "Binomial Congestion Control Algorithms", in IEEE INFOCOM 2001

[7] Fernando Paganini, Zhikui Wang, John C. Doyle and Steven H. Low, " Congestion Control for High Performance, Stability, and Fairness in General Networks", in IEEE/ACM Transactions On Networking, VOL. 13, NO. 1, February 2005

[8] Hyun Cheol Cho, M. Sami Fadali and Kwon Soon Lee, "Internet Traffic Control Using Dynamic Neural Network", in Journal Of Electrical Engineering and Technology, Vol. 3 No. 2. PP. 285-291, 2008

[9] Hyun C. Cho, M. Sami Fadali, Hyunjeong Lee, "Neural Network Control For TCP Network congestion", in American Control Conference, June 8-10,2005

[10] J. Alan Bivens, Boleslaw k. Szymanski, Mark J. Embrechts, "Network congestion arbitration and source problem prediction using neural networks", smart engineering system design, vol. 4:243-252, 2002

[11] Kun Tan, Feng Jiang, Qian Zhang and Xuemin Shen, "Congestion Control in Multihop Wireless Networks", in IEEE transactions on Vehicular Technology, Vol. 56. No. 2, March 2007

[12] Lijun Chen, Steven H. Low and John C. Doyle, "Joint Congestion Control and Media Access Control Design for Ad Hoc Wireless Networks" Engineering \& Applied Science Division, California Institute of Technology Pasadena, CA 91125, 2004

[13] Lisong Xu, Khaled Harfoush, and Injong Rhee, "Binary Increase Congestion Control (BIC) for Fast LongDistance Networks", in IEEE INFOCOM 2004

[14] L. Khoukhi ,S. Cherkaoui “ Intelligent Solution For Congestion Control In Wireless Ad Hoc Networks"
Department of electrical and Computer Engineering, University Of Sherbrooke JIK 2RI,QC, Canada 2003

[15] Mehdi Mohtashamzadeh and Mehdi Harizi, "Multi-Part Congestion Control algorithm For TCP Networks using Neural Networks", in International Journal on Recent Trends In Engineering and Technology, Vol. 7, No. 1, July 2012.

[16] Melinda Barbas, Georgeta Boanea and Virgil Dobrota "Multipath Routing Management using Neural Network Based Traffic Prediction “ Emerging 2011: The Third International Conference On Emerging Network Intelligence ,ISBN: 978-1-61208-174-8, 2011.

[17] N. kojic, I.Reljin and B. Reljin, "Neural network based Dynamic Multicast Routing", Elektronika Ir Elektrtechnika, ISSN 1392-1215, Vol. 19, No. 3,2013

[18] Nenad Kojic ,Irini Reljin and Branimir Reljin,’Neural Network for Optimization of Routing in Communication Networks", Facta Universitatis (NIS),SER.: Elec.Energ. vol. 19, no. 2,August 2006, 317-329

[19] P.Rama Devi and Dr .D. Srinivasa Rao, "Congestion Adaptive Hybrid Multi-Path Routing Protocol For Load Balancing in Mobile Ad hoc Networks", in International Journal of Computer Science and Telecommunications Vol. 3,issue 12 December 2012

[20] Pallapa Venkataram,Sudip Ghosal and B.P. Vijay Kumar, "Neural network based optimal algorithm for communication networks", Protocol Engineering and Technology (PET) unit, Department of Electrical communication Engineering, Indian Institute of Science, Bangalore India, 17 May 2002

[21] Saverio Mascolo, "Congestion Control in High Speed Communication network using the Smith Principle" ,Dipartimento di Elettrotecnica ed Elettronica , Politecnico di Bari Via Orabona 4,70125 Bari,Italy,14 March.1999

[22] Yen Chieh Ouyang, Ching-Wen Yang and Wei Shi Lian, " Neural Network based variable Bit rate Traffic Prediction for Traffic Control using Multiple Leaky Bucket",in Journal Of high speed Networks , 2006.

[23] http://slideshow.techworld.com/3363475/ipv6--why-weneed-new-internet-protocol/8/ 27 June 2014

[24] http://www.techspot.com/news/52011-one-minute-onthe-internet-640tb-data-transferred-100k-tweets-204million-e-mails-sent.html 27 June 2014 